FEDSM2012-72266

\title{
CALIBRATION OF THE NASA GRC 16" MASS-FLOW PLUG
}

\author{
David O. Davis \\ NASA Glenn Research Center \\ Cleveland, $\mathrm{OH}$, USA
}

\author{
J. David Saunders \\ NASA Glenn Research Center \\ Cleveland, $\mathrm{OH}, \mathrm{USA}$
}

\author{
Franco C. Frate \\ ASRC Aerospace Corp \\ Cleveland, OH, USA
}

\author{
David J. Friedlander \\ NASA Glenn Research Center \\ Cleveland, $\mathrm{OH}, \mathrm{USA}$
}

\section{ABSTRACT}

The results of an experimental calibration of the NASA Glenn Research Center 16" Mass-Flow Plug (MFP) are presented and compared to a previously obtained calibration of a 15" Mass-Flow Plug. An ASME low-beta, long-radius nozzle was used as the calibration reference. The discharge coefficient for the ASME nozzle was obtained by numerically simulating the flow through the nozzle from the WIND-US code. The results showed agreement between the 15 " and 16" MFPs for area ratios (MFP to pipe area ratio) greater than 0.6 but deviate at area ratios below this value for reasons that are not fully understood. A general uncertainty analysis was also performed and indicates that large uncertainties in the calibration are present for low MFP area ratios.

\section{INTRODUCTION}

Large-scale model supersonic aircraft inlets are routinely tested in NASA Glenn Research Center's (GRC) larger wind tunnels such as the $8 \times 6 \mathrm{ft}$ and $10 \times 10 \mathrm{ft}$ Supersonic Wind Tunnels (SWT). Two key objectives of such tests are to ensure that the air supplied by an inlet matches the mass-flow demand of an engine and that the inlet operates in a stable condition over a wide range of mass-flows. Thus, accurate measurement of inlet mass-flow is of primary importance. Due to its relatively simple mechanical design, the mass flow through an inlet is often controlled and measured by a calibrated conical mass-flow plug (MFP). For larger models, NASA GRC uses a 16" diameter MFP which was fabricated by modifying a pre-existing 15" MFP. Calibration of the 16" MFP plug was performed in-house using an ASME low-beta, long-radius nozzle as the reference measurement. The procedure and resulting calibration, as well as a comparison to the calibration of the previous 15" MFP are reported herein.

\section{NOMENCLATURE}

$A \quad=$ Area $\left(\mathrm{in}^{2}\right)$

$A R=$ MFP area to cold-pipe area ratio (Eq. 20)

$C_{D} \quad=$ Discharge coefficient $d \quad=$ ASME nozzle diameter (in)

$\mathrm{D} \quad=$ Diameter (in)

$g_{c} \quad=$ Proportionality constant $\left(32.17405 \mathrm{lb} \cdot \mathrm{ft} /\left(\mathrm{lbf} \cdot \mathrm{s}^{2}\right)\right.$

$L_{1} \quad=$ ASME nozzle throat length (in, Figure 3)

$M \quad=$ Mach number

MFP $\quad=$ Mass Flow Plug

$p \quad=$ Pressure $(\mathrm{psi})$

$R \quad=$ Radius (in)

$R_{1} \quad=$ ASME nozzle ellipse major radius (in, Figure 3 )

$R_{2} \quad=$ ASME nozzle ellipse minor radius (in, Figure 3 )

$R_{\text {air }} \quad=$ Gas constant for air $(53.3525 \mathrm{ft} \cdot \mathrm{lbf} /(\mathrm{lb} \cdot \mathrm{R})$

$\operatorname{Re}_{d} \quad=$ ASME nozzle Reynolds number

$\operatorname{Re}_{d, s} \quad=$ Scaled ASME nozzle Reynolds number $\operatorname{Re}_{d, s}=\operatorname{Re}_{d} \times 10 \mathrm{E}-06$

$=$ Slant height of the frustum of a cone (in, Figure 5)

$=$ Temperature $(\mathrm{R})$ or throat tap location (in, Figure 3 )

$=$ Velocity $(\mathrm{ft} / \mathrm{sec})$

$=$ mass flow rate $(\mathrm{lb} / \mathrm{s})$

$=$ Cartesian coordinates

$=$ Cylindrical coordinates

$=$ Measured position of MFP (in)

$=$ Effective position of MFP (in, Figure 5 and Eq. 23)

Greek Symbols

$\beta \quad=$ Ratio of ASME nozzle-to-approach pipe diameter

$\gamma \quad=$ Ratio of specific heats

$\delta X_{i} \quad=$ Uncertainty of measurand $X_{i}$

$\rho \quad=$ Density $\left(\mathrm{lb} / \mathrm{ft}^{3}\right)$

$\phi \quad=$ MFP cone half-angle (deg, Figure 5)

Subscripts

$a \quad=$ Pertaining to the average component

amb = Pertaining to ambient conditions

$\delta^{*}=\quad=$ Pertaining to boundary-layer blockage (displacement thickness)

$i \quad=$ Pertaining to ideal conditions

$n \quad=$ Pertaining to the normal component

This material is declared a work of the U.S. Government and is not subject to copyright protection in the United States. Approved for public release; distribution is unlimited. 


$$
\begin{array}{ll}
\text { noz } & =\text { Pertaining to the ASME nozzle } \\
\text { pipe } & =\text { Pertaining to the cold-pipe } \\
\text { plug } & =\text { Pertaining to the MFP } \\
\text { pot } & =\text { Pertaining to potential flow conditions } \\
t & =\text { Pertaining to total conditions } \\
v & =\text { Pertaining to the vertical component }
\end{array}
$$

Superscripts

$$
\text { * } \quad=\text { Pertaining to choked flow conditions }
$$

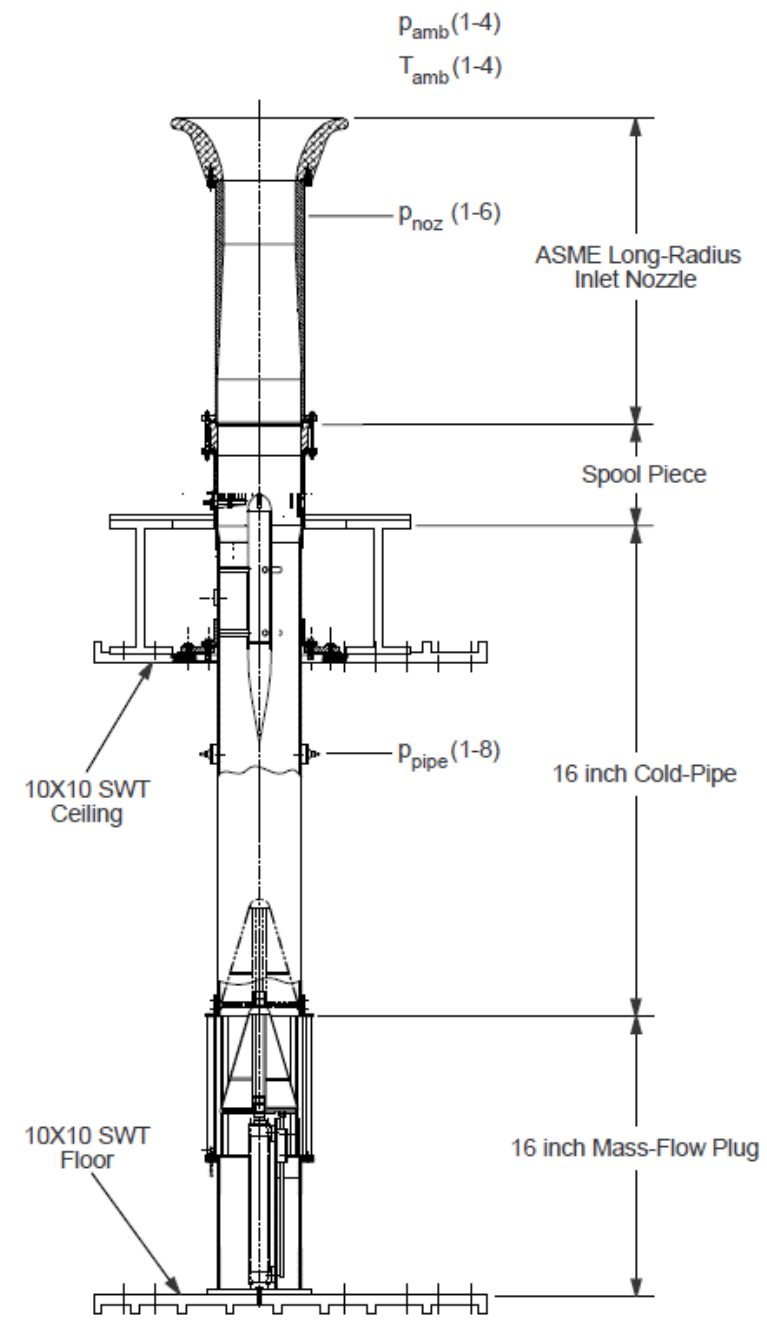

Figure 1. Schematic of 16" MFP Setup in 10x10ft SWT.

\section{CALIBRATION PROCEDURE}

The calibration of both the caged 15" and 16" mass-flow plugs were carried out with the experimental setup shown in Figure 1. The four primary components of the calibration rig are the American Society of Mechanical Engineers (ASME) inlet flow nozzle assembly, an adapter spool piece, the cold-pipe assembly, and the mass-flow plug assembly. The entire calibration assembly was mounted vertically on the floor of NASA Glenn's 10x10-ft Supersonic Wind Tunnel and protruded through a sealed opening in the ceiling. The ASME nozzle was fed by atmospheric ambient pressure and temperature air while the wind tunnel exhausters were used as a vacuum source to establish a flow through the assembly. A photo of the ASME nozzle on the top of the $10 \times 10-\mathrm{ft}$. SWT is shown in Figure 2 and a schematic of the geometry is shown in Figure 3. A photo of the 16" MFP attached to the cold-pipe is shown in Figure 4 and a schematic of the plug is shown in Figure 5 .

The spool piece is an adapter to match the ASME nozzle exit diameter to the cold-pipe diameter under test. The cold-pipe is simply a 96" long constant-area pipe whose primary purpose is to allow for a flow-settling length between the inlet exit and the mass-flow plug. Near the forward end is a spherical-nosed centerbody supported by three equally spaced struts. The centerbody nose is intended to simulate the fan spinner on a turbojet engine. Attached to the forward end of the cold-pipe is a spool piece that houses eight compressor face rakes. The rakes lie in the plane of the tip of the centerbody nose. The inside edge at the exit plane of the cold-pipe is sharp to fix the position of the mass-flow plug choke point.

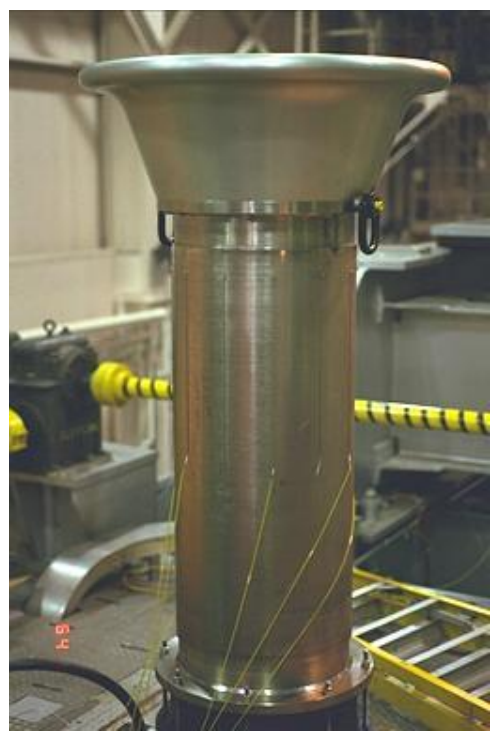

Figure 2. ASME nozzle assembly.

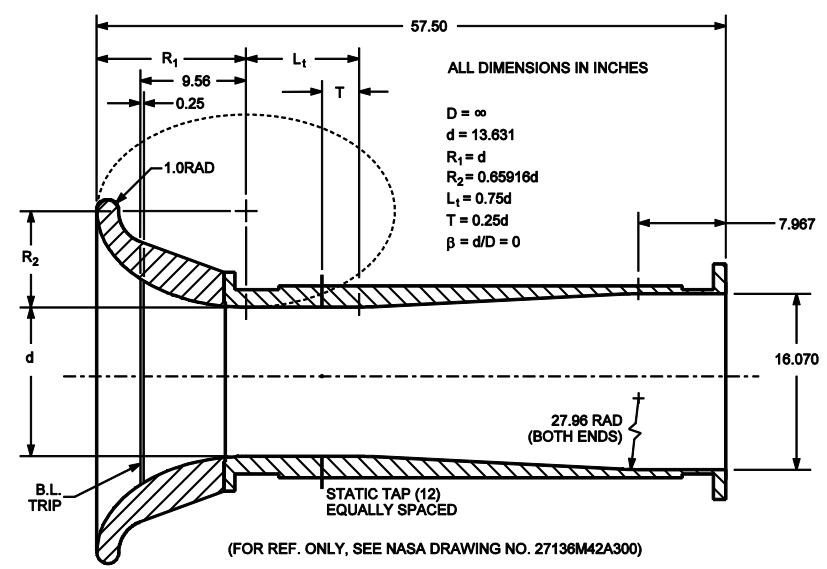

Figure 3. ASME nozzle schematic.

A picture of the 16-inch mass-flow plug attached to the cold-pipe is shown in Figure 4. The cone is actuated by a hydraulic cylinder and concentricity of the cone with the cold-pipe is maintained by wedge bearings on three of the six knife-edged support struts (cage). A position transducer (potentiometer) can be seen on the top of the assembly in Figure 4. 


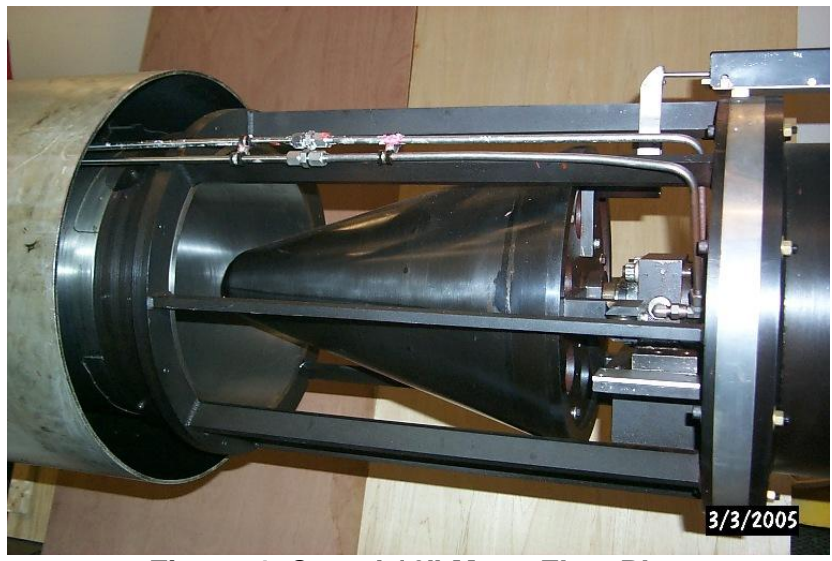

Figure 4. Caged 16" Mass-Flow Plug.

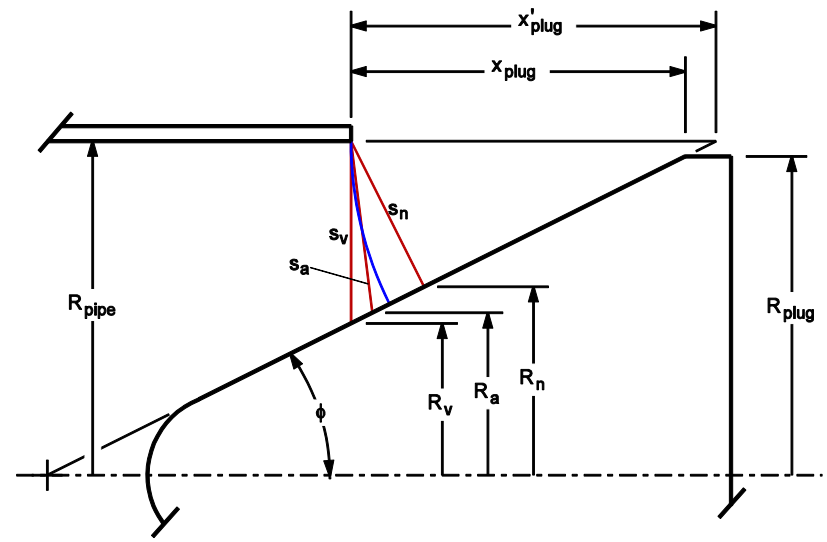

Figure 5. Schematic for MFP sonic (choked) area.

The ultimate goal of the calibration is to determine the discharge coefficient for the 16" MFP over an operating range typical of the supersonic inlet testing. The discharge coefficient for the mass-flow plug is defined as:

$$
\text { Eq. } 1 \quad C_{D, p l u g}=\frac{w_{\text {noz }}}{w_{\text {plug }, i}^{*}}
$$

where $w_{n o z}$ is the actual mass flow through the cold-pipe and $w_{p l u g, i}^{*}$ is the ideal inviscid choked flow through the mass-flow plug. For the purpose of calibrating the mass-flow plug, the actual mass flow was measured with an ASME Long-Radius Flow Nozzle. A description of this nozzle is as follows.

\section{ASME Nozzle}

Geometry: An ASME Long-Radius Flow Nozzle with throat taps was used to provide a reference mass-flow measure for calibrating both the 15 " and 16" mass-flow plugs. The geometry of the nozzle is shown in Figure 3. This design conforms to the Low- $\beta$ Nozzle with Throat Taps illustrated in Fig. II-III-14 of Ref. 2 with the following two exceptions. First, no approach pipe exists before the nozzle $(D=\infty)$, hence $\beta=d / D=0$. And second, the nozzle exit flow does not exhaust into a sudden expansion but rather into a shallow angle conical diffuser. The nozzle throat-to-conical diffuser and conical diffuser-toconstant area end section are both transitioned by 27.96 " radii. $^{1}$

Citing the desire to maintain fully turbulent flow in the nozzle, the calibration tests were performed both with and without a boundarylayer trip in the inlet region of the nozzle. Details of the trip were not supplied by Iek [1] but inspection of the ASME nozzle indicated the trip location and width are as shown in Figure 3. Most of the tests were performed with an 80 grit trip, but limited testing was also performed with a 36 grit trip with essentially the same results.

Flow Equations: The flow through the ASME nozzle is calculated by the following equation:

Eq. 2

$$
w_{n o z}=C_{D, n o z} \cdot w_{n o z, i}
$$

where

Eq. 3

$$
w_{n o z, i}=\rho_{n o z} \cdot U_{n o z} \cdot A_{n o z}
$$

and where $\rho_{n o z}$ and $U_{n o z}$ are evaluated at the throat of the ASME nozzle. Substitution of the ideal gas law, Mach number definition, and isentropic and adiabatic relations for pressure and temperature, respectively:

Eq. 4

$$
\rho_{n o z}=\frac{144 \cdot p_{n o z}}{R_{a i r} \cdot T_{n o z}}
$$

Eq. 5

$$
U_{n o z}=M_{n o z} \cdot \sqrt{\gamma \cdot g_{c} \cdot R_{a i r} \cdot T_{n o z}}
$$

Eq. 6

$$
p_{n o z}=\frac{p_{t, n o z}}{\left(1+\frac{\gamma-1}{2} \cdot M_{n o z}^{2}\right)^{\frac{\gamma}{\gamma-1}}}
$$

Eq. 7

$$
T_{n o z}=\frac{T_{t, n o z}}{\left(1+\frac{\gamma-1}{2} \cdot M_{n o z}^{2}\right)}
$$

into the expression for $w_{0}$ and rearranging yields:

Eq. 8

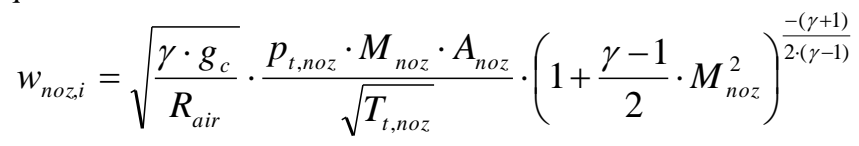

Substituting the values for $\gamma, g_{c}$ and $R_{\text {air }}$ and replacing $p_{t, n o z}$ and $T_{t, n o z}$ with $p_{a m b}$ and $T_{a m b}$, respectively, gives the final form:

Eq. $9 w_{n o z, i}=0.91884 \cdot \frac{p_{a m b} \cdot M_{n o z} \cdot A_{n o z}}{\sqrt{T_{a m b}}} \cdot\left(1+0.2 \cdot M_{n o z}^{2}\right)^{-3}$

\footnotetext{
${ }^{1}$ Note that this diffuser geometry differs from the description given by Iek [1] where the diffuser is described as being a "cubic contour."
} 
where $M_{n o z}$ is the Mach number of the flow at the throat of the nozzle and is given by the isentropic relation:

Eq. $10 \quad M_{n o z}=\sqrt{5 \cdot\left(\left(\frac{p_{a m b}}{p_{n o z}}\right)^{\left(\frac{1}{3.5}\right)}-1\right)}$

and $p_{n o z}$ is the static pressure measured at the throat of the nozzle. All of the variables in Eq. 9 and Eq. 10 are directly measured or calculated from directly measured quantities. The only remaining parameter required to calculate the nozzle flow rate is the nozzle discharge coefficient $\left(C_{D, \text { noz }}\right)$.

\section{ASME Nozzle Discharge Coefficient}

Historical Correlation: The discharge coefficient for a nozzle with throat taps is a function of the potential flow nonuniformity and the boundary-layer displacement thickness resulting from viscous effects. As discussed by Rivas and Shapiro [3], if the length of the cylindrical sections before and after the throat taps are on the order of or greater than $1 / 4$ throat diameter, then the effects of potential flow non-uniformity are negligibly small.

In the absence of a calibration, ASME recommends reading the discharge coefficient for a low- $\beta$ long-radius nozzle with throat taps from Fig. II-III-19 of Ref. [2]. The source of this curve is not referenced but it appears to be the "Calculated Curve" in Fig. 22 of Cotton and Westcott [4]. No uncertainty is given for using this curve but based on similar analysis, it is likely to be on the order of \pm 1.5 $2.0 \%$. Much of the data used to generate this curve was based on incompressible flow data. In 1973, Smith and Matz [5] investigated an 8 -inch low- $\beta$, long-radius nozzle under incompressible and compressible conditions and reported a Mach number dependence.

Two-Code Computational Approach: For the March 1996 calibration of the 15" caged mass-flow plug [1] due to the uncertain effect of the $\beta$ parameter and the nozzle exit diffuser on the discharge coefficient, the ASME correlation was not used. Instead, the theoretical method first performed by Smith and Matz [5] and later by Lahti and Hamed [6] was used to establish a discharge coefficient. With this method, the discharge coefficient is assumed to depend on two components, an inviscid part $\left(C_{D, p o t}\right)$ that accounts for the radial pressure variation in the throat, and a viscous part $\left(C_{D, \delta^{*}}\right)$ that accounts for the effective boundary-layer blockage. Computer programs were used to calculate each component and the discharge coefficient of the nozzle was determined from the product of the components:

$$
\text { Eq. } 11 \quad C_{D, n o z, 2 C}=C_{D, p o t} \cdot C_{D, \delta^{*}}
$$

This approach will hereafter be referred to as the "Two-Code" (2C) nozzle calibration. In Lahti and Hamed's [6] study, the potential solution was obtained with the Stream Tube Curvature (STC) computer code [7], chosen because of their many years of experience with it and because it is driven by global mass conservation as part of its solution procedure making it a natural choice for mass-flow calibration studies. The boundary-layer correction was obtained using Harris and Blanchard's code [8,9]. This code was chosen based on its wide use and thorough documentation. Lahti and Hamed [6] report that comparisons with experimentally determined discharge coefficients indicate that an accuracy of $\pm 0.25 \%$ can be achieved with their method.
For the calibration of the NASA Glenn ASME nozzle, Iek [1] followed a similar approach but used different computer codes. For the potential solution, Stockman and Farrell's code [10] was used and for the viscous solution, Herring's PL2 code [11] was used. With these codes, the theoretical discharge coefficient for the ASME nozzle was determined at six discrete throat Reynolds numbers.

Table 1. Discharge Coefficient vs. Reynolds Number

\begin{tabular}{|c|c|}
\hline $\operatorname{Re}_{d, s}=\mathrm{Re}_{d} \times 10^{-6}$ & $C_{D, \text { noz }}$ \\
\hline \hline 1.6828 & 0.98553 \\
\hline 3.0386 & 0.98667 \\
\hline 3.7659 & 0.98728 \\
\hline 4.0302 & 0.98728 \\
\hline 4.3062 & 0.98755 \\
\hline 4.6056 & 0.98796 \\
\hline
\end{tabular}

For data reduction during the mass-flow plug calibrations, the data in Table 1 were interpolated with a third-order polynomial function: ${ }^{2}$

Eq. 12

$$
\begin{aligned}
& C_{D, \text { noz, } 2 C}=0.982982+0.002081573 \mathrm{Re}_{d, s}- \\
& 0.0004063900 \mathrm{Re}_{d, s}^{2}+0.00004062582 \mathrm{Re}_{d, s}^{3}
\end{aligned}
$$

and extrapolated by setting points outside the calibration range to the end points of the calibration.

RANS Computational Approach: More recently, a different computational approach was taken for obtaining the discharge coefficient of the ASME nozzle as well as the 16" MFP. Rather than use a potential code coupled with a boundary-layer calculation, WIND-US, which is a general purpose 3-D Reynolds-averaged Navier-Stokes (RANS) code, was used for the numerical studies [12]. Versions 3 Alpha and 2 of WIND-US calculated the CFD derived discharge coefficients. WIND-US is a mature, multi-zone, structuredgrid, compressible flow solver offering a variety of turbulence models. All simulations were run steady-state using the Menter Shear Stress Transport (SST) turbulence model [13]. The SST turbulence model was chosen for all of the simulations because previous studies $[14,15]$ have shown that this particular model performs well for jet flows.

An axisymmetric structured grid, composed of roughly 119,000 grid points and divided into 17 zones, was used to model the test rig assembly shown in Figure 1. Grids were created for 19 different plug positions, ranging from $x_{p l u g}=2.4$ " to 16.4 " with $x_{p l u g}$ defined as shown in Figure 5. Zones upstream of the experimental boundary-layer trip location (see Figure 3) assumed laminar flow while zones downstream of the trip location assumed turbulent flow. An example grid is shown in Figure 6 while Figure 7 illustrates the solid computer model.

The simulations used a constant Courant-Friedrichs-Lewy (CFL) number of 0.5 rather than a constant time step. To reduce computational costs and convergence time, parallel processing along with three levels of grid sequencing were used to obtain converged solutions. First, a coarse grid sequence included only every fourth point in each direction. Second, a medium grid sequence was used that utilized every other point in each direction. Third, a fine sequence used every grid point. Solution convergence was achieved when the computational equation residuals were reduced by several orders of magnitude and the mass flow rates in the bellmouth entrance and near

\footnotetext{
${ }^{2}$ Ref. 1 indicates a fourth-order polynomial was used but the actual data reduction program uses the third-order fit shown.
} 
the cold pipe exit each varied by less than $0.09 \%$ per iteration. Convergence was typically obtained in a little over three hours.

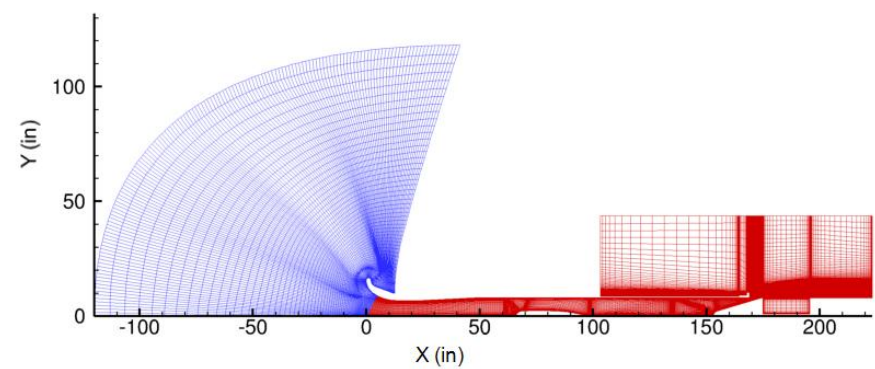

Figure 6. Sample primary grid layout, laminar zones are in blue, turbulent zones are in red.

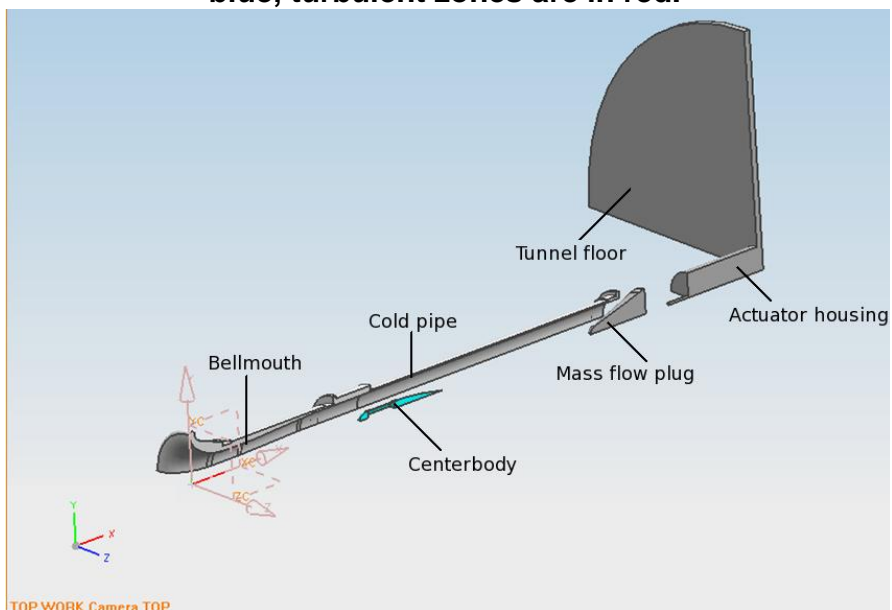

Figure $7.90^{\circ}$ sector of the axisymmetric solid model.

Initially, the WIND-US code was run with the as-tested ambient inlet conditions $\left(p_{a m b}=14.3 \mathrm{psia}\right)$, but then to better resolve Reynolds number effects, additional cases were run at inlet pressures of 5.0 and 50.0 psia. For all pressures, the ambient total temperature was set to $520.0^{\circ} \mathrm{R}$ with an initial inflow Mach number set to 0.001 . Whereas for the 5.0 psia case, the back-pressure at the MFP exit was set at 1.0 psia, for the two higher pressure cases, the back-pressure was set at the astested pressure of 2.0 psia.

After the CFD analyses were completed, the data was postprocessed to acquire the discharge coefficients for the bellmouth nozzle. First, the mass flow rate through the cold pipe was calculated from the average mass flow rate between the downstream tangential edge of the radial nose of the conical plug to the cold pipe exit:

Eq. 13

$$
w_{\text {pipe }}=\frac{\sum_{i=1}^{N} w_{\text {pipe } x, i}}{N}
$$

where

Eq. 14

$$
w_{\text {pipe } x}=\int_{\phi} \int_{r} \rho \cdot U_{x} \cdot r \cdot d r \cdot d \theta
$$

This was compared to the average mass flow rate at the throat of the bellmouth to check for continuity. The solution was converged when the average mass flow rates in the bellmouth throat and just upstream of the cold pipe exit varied by less than $0.09 \%$ of each other. The discharge coefficient could then be calculated from:
Eq. 15

$$
C_{D, n o z}=\frac{w_{\text {pipe }}}{w_{n o z, i}}
$$

where $w_{n o z, i}$ is given by Eq. 9 .

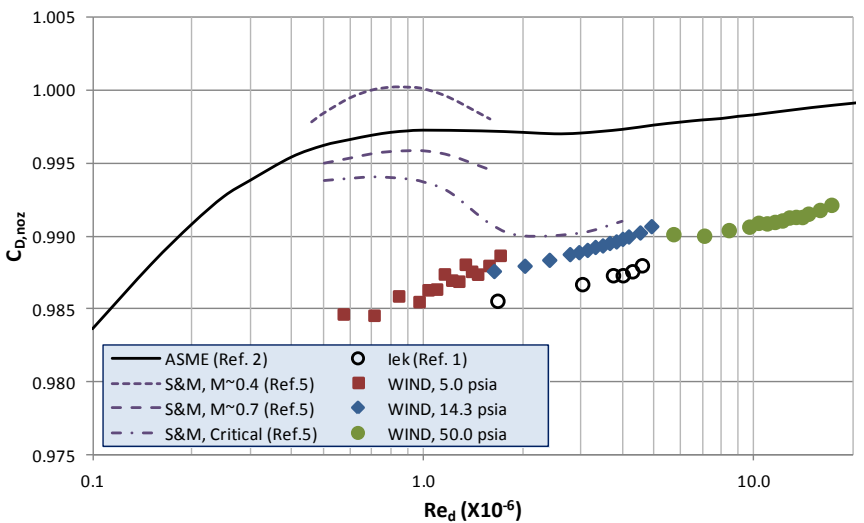

Figure 8. ASME nozzle discharge coefficient

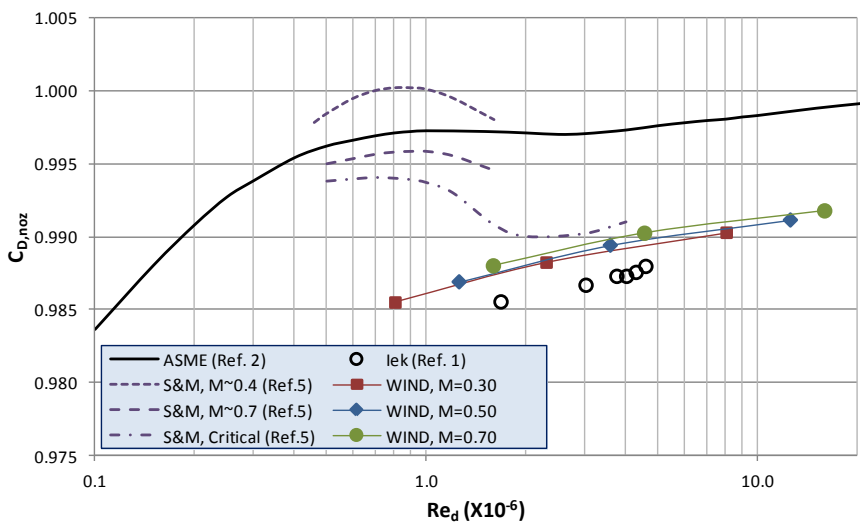

Figure 9. ASME nozzle discharge coefficient, constant Mach number.

The data from the WIND-US computations are plotted in Figure 8. For reference, the ASME curve, the data from Smith and Matz, and the numerical data from Iek are also shown. For the WIND-US data, only points where the throat Mach number was between 0.2 and 0.8 are plotted. Due to the use of a boundary-layer trip on the nozzle, the distribution does not display the transitional behavior of Smith and Matz [5] data and indeed seems to agree well with the turbulent portion of their "Critical" curve $\left(\operatorname{Re}_{d, n o z}>2.0 \mathrm{E}+06\right)$. Another observation of the current data is the saw-tooth nature of the combined distributions indicating a small Mach number dependence. To illustrate this dependence more clearly, data at constant Mach numbers of 0.3 , 0.5 and 0.7 were extracted and are plotted in Figure 9. Although the dependence on Mach number is small for the current data, the trend is opposite that of Smith and Matz [5], but since their low Mach number data was in the transition region, nothing firm can be drawn from this. Comparing the present WIND-US data to the previous distribution established for the same nozzle configuration by Iek [1] shows that the present data trends about $0.2 \%$ higher than Iek's.

In order to apply easily the present data to the 16" MFP calibration, a correlation was developed that fits the data and accounts for the small Mach number dependence. This was accomplished by first curve fitting the $\mathrm{M}=0.5$ data which resulted in the following expression: 
Eq. 16

$$
C_{D, M=0.5}=0.993003-\frac{6.73896}{\sqrt{\operatorname{Re}}_{d}}
$$

An empirical correction to this nominal discharge coefficient was established such that the Mach number dependant discharge coefficient is defined by:

Eq. 17

$$
C_{D, n o z, W U}=C_{D, M=0.5} \cdot f(M)
$$

where

$$
\text { Eq. } 18 \quad f(M)=0.999514 \cdot\left(1-0.2 M_{n o z}^{2}\right)^{-0.0086341 \text { s }}
$$

Since it is unlikely that the current ASME nozzle would ever be operated at anything but ambient pressure conditions, the coefficients in Eq. 18 were determined from the 14.3 psia data only. A comparison of Eq. 17 and Eq. 16 with the numerical data is shown in Figure 10a and Figure 10b, respectively.

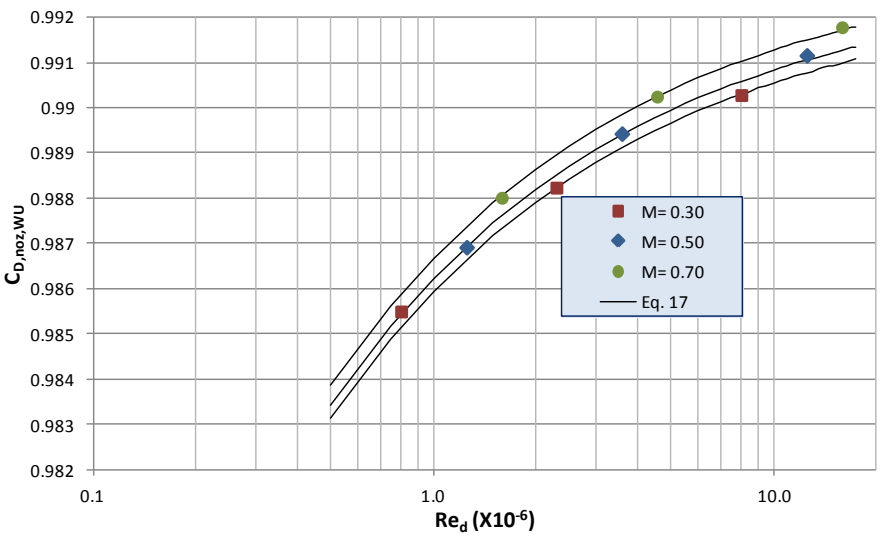

a) Fit of constant Mach number data.

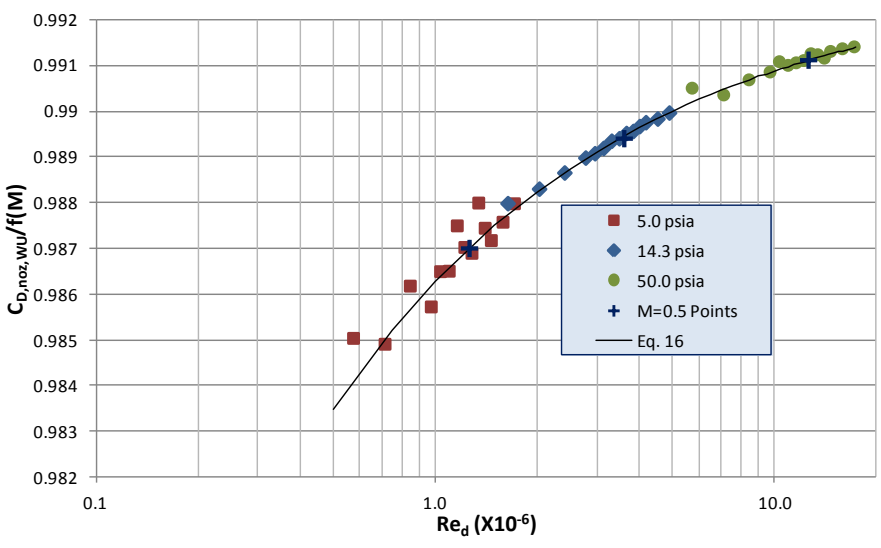

b) Fit of all data.

Figure 10. Comparison of ASME discharge coefficient correlation with numerical data.

\section{6" Mass-Flow Plug}

Geometry: With reference to Figure 5, the plug itself is a $\phi=16.5$ degree half-angle cone with a 2.18 inch nose radius. The downstream end of the cone is $R_{\text {plug }}=15.800$ inches in diameter followed by a 0.610 inch wide cylindrical shoulder.

Flow Equations: The discharge coefficient for the mass-flow plug is assumed to be a function only of the plug position and defined as the ratio of the actual mass-flow to the ideal theoretical mass-flow (see Eq. (1)). The ideal mass-flow is calculated from the following equation:

Eq. $19 \quad w_{\text {plug }, i}^{*}=\rho_{\text {pipe }} \cdot U_{\text {pipe }} \cdot A R^{-1} \cdot A_{\text {plug }}$

where

Eq. $20 \quad A R=\frac{A_{\text {plug }}}{A_{\text {pipe }}}$

and where $A_{\text {plug }}$ is the choked-flow area between the cold-pipe exit plane edge and the conical plug. With reference to Figure 5, the definition of the choked area $\left(A_{\text {plug }}\right)$ of the MFP is based on the area of the frustum of a right circular cone where the slant height $\left(s_{a}\right)$ is defined as the average of the vertical distance from the cold-pipe edge to the MFP and the slant height of a cone whose slant height is normal to the MFP surface.

Eq. 21

$$
A_{\text {plug }}=\pi \cdot s_{a} \cdot\left(R_{\text {pipe }}+R_{a}\right)
$$

where (see Figure 5)

Eq. 22

$$
\begin{aligned}
& s_{n}=x_{\text {plug }}^{\prime} \cdot \sin (\phi) \\
& s_{v}=x_{\text {plug }}^{\prime} \cdot \tan (\phi) \\
& s_{a}=\left(s_{n}+s_{v}\right) / 2.0 \\
& R_{a}=R_{\text {pipe }}-s_{a} \cdot \cos \left(\phi-\arccos \left(s_{n} / s_{a}\right)\right)
\end{aligned}
$$

and

Eq. 23

$$
x_{\text {plug }}^{\prime}=x_{\text {plug }}+\left(R_{\text {pipe }}-R_{\text {plug }}\right) / \tan (\phi)
$$

where $x_{\text {plug }}^{\prime}$ is the distance from the end of the cold-pipe to the theoretical intersection line of the plug conical surface and the cylinder of the cold-pipe internal diameter (see Figure 5). The choice of $s_{a}$ to approximate the sonic line (curved line in Figure 5) is arbitrary but must be used consistently for the calibration to be valid.

The area ratio can be expressed in terms of the Mach number in the pipe by the following isentropic relation:

Eq. 24

$$
\frac{A_{\text {plug }}}{A_{\text {pipe }}}=\left(\frac{\gamma+1}{2}\right)^{\frac{\gamma+1}{2(\gamma-1)}} \cdot M_{\text {pipe }} \cdot\left(1+\frac{\gamma-1}{2} \cdot M_{\text {pipe }}^{2}\right)^{\frac{-(\gamma+1)}{2(\gamma-1)}}
$$

Substituting Eq. 24 and Eq. 4 through Eq. 7 (with the subscript "noz" replaced with "pipe") into Eq. 19 yields:

Eq. 25

$$
w_{i, p l u g}^{*}=\sqrt{\frac{\gamma \cdot g_{c}}{R_{a i r}}} \cdot\left(\frac{p_{t, p i p e}}{\sqrt{T_{a m b}}}\right) \cdot A_{p l u g} \cdot\left(\frac{\gamma+1}{2}\right)^{\frac{-(\gamma+1)}{2(\gamma-1)}}
$$

The total pressure in the cold-pipe $\left(p_{t, p i p e}\right)$ is calculated from Eq. 6 (with the subscript "noz" replaced with "pipe"). The static pressure in the cold-pipe $\left(p_{\text {pipe }}\right)$ is directly measured with static pressure taps and 
the Mach number in the cold-pipe $\left(M_{\text {pipe }}\right)$ is calculated from Eq. 24 which assumes that the flow at the mass-flow plug exit is choked.

Instrumentation: With reference to Figure 1, the test setup was instrumented as follows. The ambient air pressure and temperature were each recorded with four transducers which were then averaged to obtain $p_{a m b}$ and $T_{a m b}$. There were a total of six throat taps on the ASME nozzle and eight static taps at the mid-point of the cold pipe. These were also averaged to obtain $p_{n o z}$ and $p_{\text {pipe }}$. The temperatures were measured with Type-E thermocouples and the pressures were measured with ESP 8400R 0-15psia modules. The data was recorded on the NASA GRC ESCORT data acquisition system.

Calibration Runs: For the caged 16" mass-flow plug calibration runs, there were six sweeps of the mass flow plug. The first three were performed with the boundary-layer trip installed on the ASME nozzle and the last three were performed with the trip removed.

\section{Uncertainty Considerations}

A general uncertainty analysis was performed for both the 15 " and 16" MFP calibrations. The uncertainty in the MFP discharge coefficients was estimated by:

Eq. $26 \quad \delta C_{D, p l u g}=\sqrt{\sum_{i=1}^{J}\left(\frac{\partial C_{D, p l u g}}{\partial X_{i}} \cdot \delta X_{i}\right)^{2}}$

where $X_{i}$ are the measurands which are summarized with their assumed uncertainty in Table 2.

Table 2 Measurand Uncertainty

\begin{tabular}{|c|c|c|c|}
\hline$i$ & $X_{i}$ & $\delta X_{i}(15 \% / 16 " \mathrm{MFP})$ & Units \\
\hline 1 & $x_{\text {plug }}$ & 0.015 & in \\
\hline 2 & $T_{\text {amb }}$ & 2.5 & ${ }^{\circ} \mathrm{R}$ \\
\hline 3 & $p_{\text {amb }}$ & $0.0060 / 0.0045$ & $\mathrm{psi}$ \\
\hline 4 & $p_{\text {noz }}$ & $0.0049 / 0.0037$ & $\mathrm{psi}$ \\
\hline 5 & $p_{\text {pipe }}$ & $0.0042 / 0.0032$ & $\mathrm{psi}$ \\
\hline 6 & $d$ & 0.0025 & in \\
\hline 7 & $D_{\text {pipe }}$ & 0.0075 & in \\
\hline 8 & $D_{\text {plug }}$ & 0.0050 & in \\
\hline 9 & $\phi$ & 0.05 & $\mathrm{deg}$ \\
\hline 10 & $C_{D, \text { noz }}$ & 0.001 & N/A \\
\hline
\end{tabular}

The measurands $P_{a m b}, P_{n o z}$ and $P_{\text {pipe }}$ are actually calculated averages of four, six and eight individual pressure measurements, respectively, and the uncertainties in Table 2 reflect the measurement redundancy. In addition, different pressure uncertainty levels were used for the 15" and 16" MFP calibrations to reflect an upgraded pressure measurement system for the 16" MFP. The measurement uncertainty for the individual modules was 0.012 and 0.009 psi for the 15 " and 16 " MFP, respectively [16]. The uncertainty in the nozzle discharge coefficient is comprised of two components. The first is related to the grid and solution convergence and is generally small $(<0.003 \%)$. The second is related to how well the CFD models the flow physics. In particular, since the nozzle discharge coefficient is primarily a function of the boundary-layer growth, the turbulence model will have an influence on the result. Further, the difference between how the boundary layer transitions on the nozzle will also affect the discharge coefficient. In the CFD, the flow is assumed laminar until the leading edge of the boundary-layer trip and fully turbulent downstream. In the experiment, there is a finite width of grit material. These effects are not easily quantifiable, thus the uncertainty specified for the nozzle discharge coefficient is to be considered a "best guess" by the authors. Finally, although the ambient temperature is measured and used in the calculations, it does not contribute to the MFP discharge coefficient uncertainty.

\section{RESULTS AND DISCUSSION}

\section{Caged 15" MFP Calibration}

The 15 " MFP was calibrated over a range from $x_{\text {plug }}=0.25$ " to 17.0" with and without distortion screens installed in the cold-pipe. A correlation was established based on the data without distortion screens. The discharge coefficient was assumed to be only a function of the choked flow area ratio (Eq. 20) and is expressed as a segmented third-order polynomial function where the coefficient values are given in Table 3:

Eq. $27 C_{D, p l u g, 2 C}=c_{0}+c_{1} \cdot A R+c_{2} \cdot A R^{2}+c_{3} \cdot A R^{3}$

Table 3. Coefficient values for caged 15" mass-flow plug.

\begin{tabular}{|c|c|c|c|}
\hline & $A R<0.203943$ & $0.203943 \leq A R<0.811515$ & $0.811515 \leq \mathrm{AR}$ \\
\hline$c_{0}$ & 1.0340732 & 0.96163863 & 1.1845804 \\
\hline$c_{1}$ & -0.58958996 & 0.0047836495 & -0.60737883 \\
\hline$c_{2}$ & 1.1527497 & -0.030427921 & 0.42665471 \\
\hline$c_{3}$ & 0 & 0.050854378 & 0 \\
\hline
\end{tabular}

This original calibration (Eq. 27) was based on the two-code ASME nozzle calibration. To update the calibration to use the more recent WIND-US based nozzle calibration, two approaches are possible. First, the data calibration data points could be recalculated with the WIND-US based nozzle calculation and a new fit established, or second, an adjustment could be applied to Eq. 27 by multiplying by the ratio of nozzle discharge coefficients:

Eq. $28 \quad C_{D, p l u g, W U}=C_{D, p l u g, 2 C} \cdot\left(C_{D, \text { noz,WU}} / C_{D, \text { noz, } 2 C}\right)$

The nozzle discharge coefficients based on both methods are plotted in Figure 11 as a function of the 15" MFP area ratio. Also plotted is the ratio of the coefficients which can be approximated by the following empirical relation:

$$
\frac{C_{D, \text { noz,WU }}}{C_{D, \text { noz, } 2 C}}=1.0014446+0.017657109 \cdot \mathrm{AR}-
$$

Eq. 29

$$
\begin{aligned}
& 0.00069852959 / \mathrm{AR}-0.046818586 \cdot \mathrm{AR}^{2}+ \\
& 2.3567944 \mathrm{E}-05 / \mathrm{AR}^{2}+0.049185331 \cdot \mathrm{AR}^{3}- \\
& 4.4263225 \mathrm{E}-07 / \mathrm{AR}^{3}-0.018204318 \cdot \mathrm{AR}^{4}
\end{aligned}
$$

The original and adjusted 15" MFP calibrations along with the data they were derived from are shown in Figure 12. The nozzle Mach number and Reynolds number are also plotted for reference. With regard to the original calibration, there are a couple of things to note about this distribution. At area ratios below approximately 0.2 , the curve has a large negative slope and at the lowest area ratio, the discharge coefficient exceeds unity with large data scatter. At the 
largest area ratio, the slope and scatter also increases. During the test, the ratio of the cold-pipe base pressure to pipe total pressure is monitored to ensure the plug is operating under choked conditions. The two highest area ratio positions are approaching flow conditions that challenge the assumption of a well-defined choked flow.

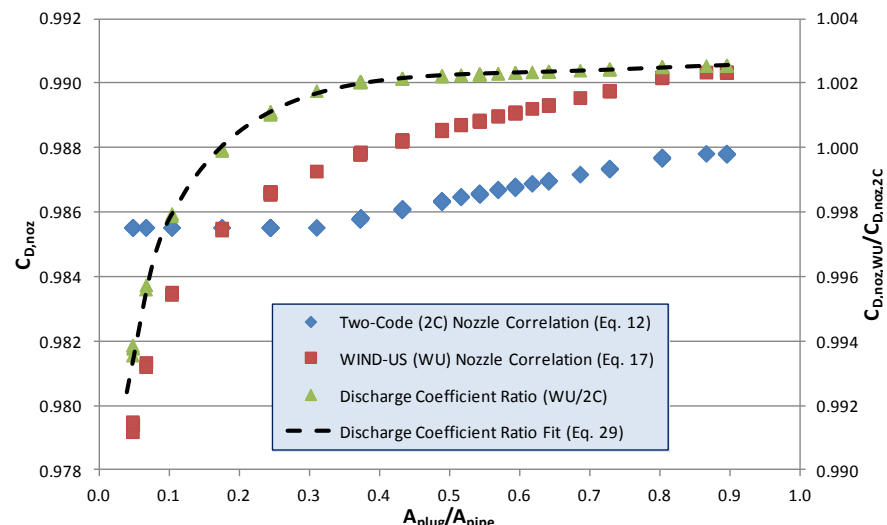

Figure 11. ASME Nozzle discharge coefficient as a function of 15" MFP area ratio.

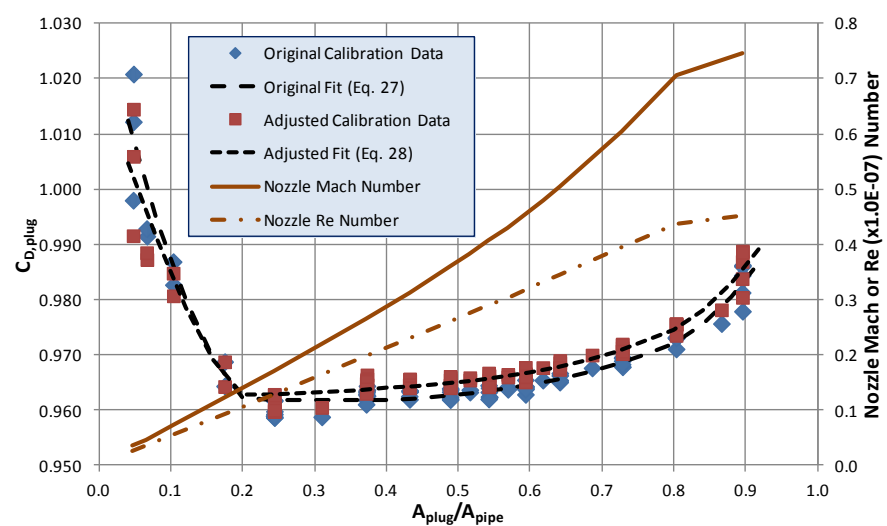

Figure 12. Original and adjusted 15" MFP discharge coefficient.

The adjusted 15" MFP discharge coefficient curve fit is re-plotted in Figure 13 with the uncertainty bounds from the general uncertainty analysis. Also plotted is the percent uncertainty based on the assumed measurand uncertainties (Table 2).

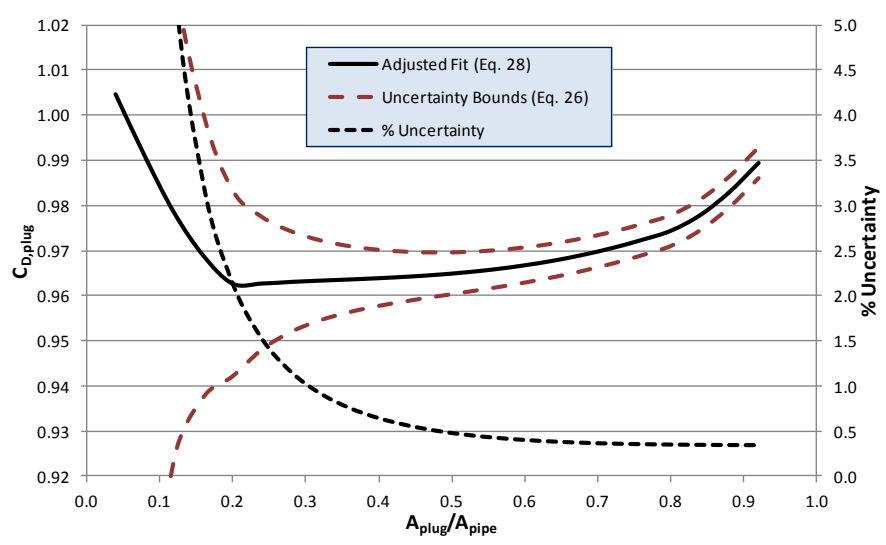

Figure 13. 15" MFP discharge coefficient uncertainty.
These results indicate that the measurement uncertainty exceeds $0.5 \%$ for MFP area ratios below 0.5 . Based on this calibration, the inlet should be sized to operate within the area ratio range $0.2<A R<0.8$ $\left(1.85\right.$ " $<x_{\text {plug }}<10.4$ ").

The relative contribution from the individual measurands is plotted in Figure 14. Also plotted for reference is the absolute uncertainty. The large uncertainty at low area ratios is primarily due to the low Mach number in the nozzle (see Figure 12) which is calculated from Eq. 10.

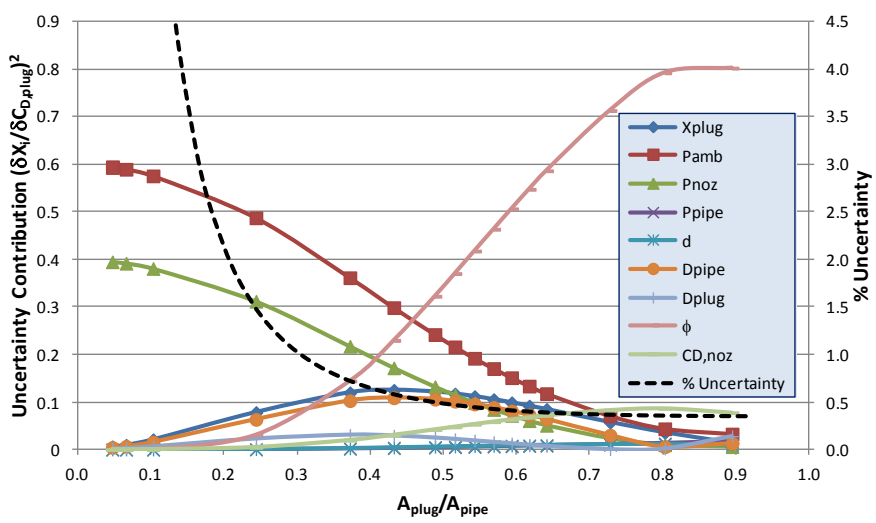

Figure 14. Measurand contribution to overall 15" MFP discharge coefficient uncertainty.

\section{Caged 16" MFP Calibration}

The results of the calibration of the 16" MFP for both tripped and un-tripped nozzle boundary layer are shown Figure 15. The nozzle Mach number and Reynolds number are also plotted for reference. At a given area ratio, due to its larger size, the 16" MFP has a larger flow rate than the 15" MFP. At large area ratios, this results in choking of the nozzle for the 16" MFP. Thus data beyond an area ratio of 0.7 will be excluded from further analysis as the MFP would be operating unchoked.

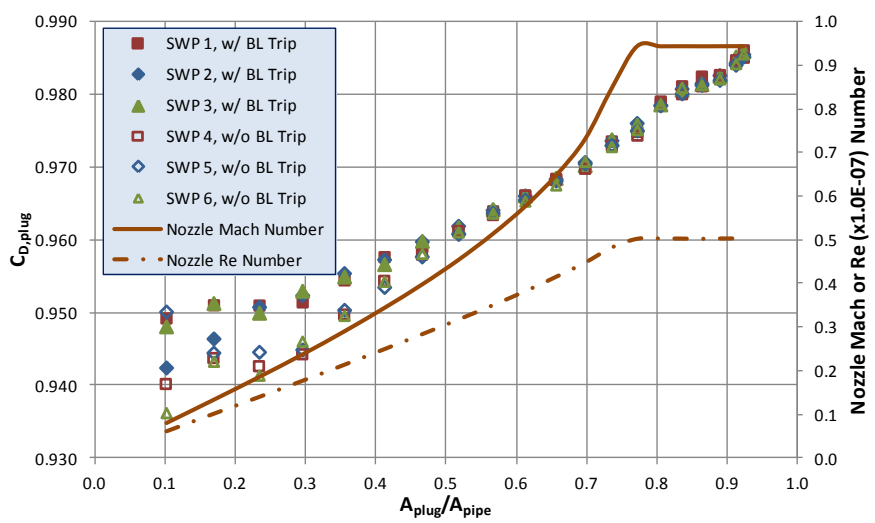

Figure 15. 16" MFP discharge coefficient data.

Comparing the 16" MFP data with and without the boundary-layer trip shows agreement above an area ratio of about 0.5 , but deviates significantly below this value. At an area ratio of 0.5 , the nozzle Reynolds number is about 3.0E+06. From Figure 9, a Reynolds number of $3.0 \mathrm{E}+06$ is consistent with the end-of-transition implied by the data of Smith and Matz [5] and the ASME curve [2]. Smith and Matz data show a large increase in discharge coefficient below this transition value. Since a fully turbulent nozzle discharge coefficient was used in the 16" MFP calibration, which was artificially low for the case with sweeps with no boundary-layer trip, the resulting MFP 
calibration is under predicted. In fact, what the transitional nozzle discharge coefficient would have been could be estimated by correcting the no-trip data to agree with the tripped data.

A comparison between the adjusted 15" MFP and the tripped 16" MFP calibration data is shown in Figure 16. The 16" MFP calibration data above an area ratio of 0.7 has been removed (ASME nozzle choked) and the remainder of the data was fit with a second order polynomial:

Eq. 30

$$
C_{D, \text { lug }}=0.943044+0.026915 \cdot \mathrm{AR}+0.017376 \cdot \mathrm{AR}^{2}
$$

Also included in this plot are the uncertainty bounds from the general uncertainty analysis. The experimentally determined discharge coefficients for the 15 " and 16" MFP tend to agree well above an area ratio of 0.6 , but deviate below this ratio. In particular, the 16" MFP curve does not exhibit the abrupt increase in flow coefficient below an area ratio of 0.2 . Inasmuch as the calibration curves lay at the edges of the others uncertainty bounds, this suggests that the differences in calibration are not fully attributable to measurement uncertainties. For both MFPs, the method of calibration produces large uncertainties at low area ratios. This is primarily due to the low Mach number in the ASME nozzle. The use of a delta pressure transducer at low Mach numbers would mitigate the large uncertainties significantly.

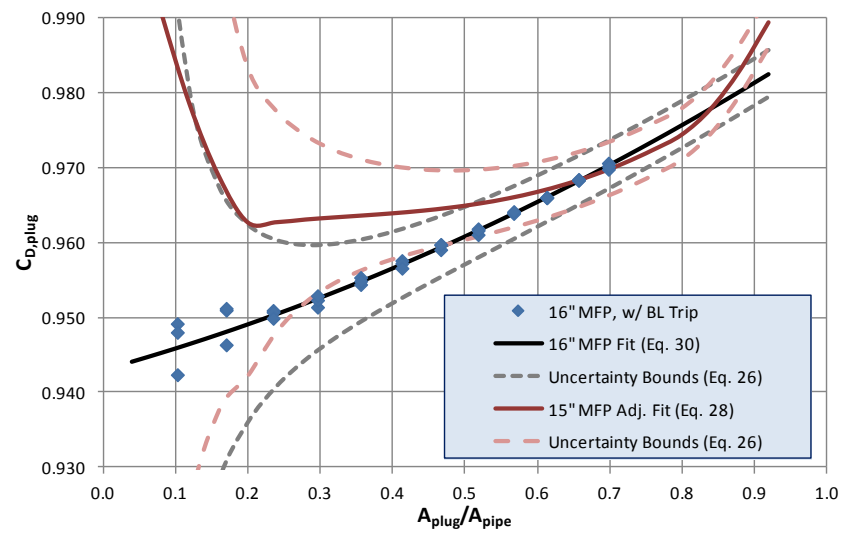

Figure 16. Comparison of 15" and 16" MFP calibrations.

As previously mentioned, the numerical study with WIND-US included a numerically based determination of the 16" MFP discharge coefficient (see Figure 6 and Figure 7). This study is still in progress and will be reported in a future publication, but the preliminary results indicate a trend very similar to the experimental 16" MFP results but shifted up about $0.25 \%$. However, results for area ratios below 0.2 have not yet been obtained so no comment can be made regarding the tail-up behavior of the 15 " MFP.

\section{CONCLUDING REMARKS}

The results of a calibration procedure for the NASA GRC 16" MFP have been presented and compared to a previous calibration of a 15 " MFP. The 16" MFP was fabricated from the 15" MFP so all future testing will be with the 16 " MFP. For the 16" MFP, a calibration has been established, but a difference in character between the 15" MFP and the 16" MFP for area ratios below 0.6 is observed. Unfortunately, this region of disagreement coincides with large uncertainty levels. The recommended path forward is to first complete the numerical calibration of the 16" MFP; in particular obtaining calibration data below an area ratio of 0.2 . And second, the experimental calibration of the 16" MFP should be repeated with a focus on minimizing measurement uncertainties.

\section{ACKNOWLEDGMENTS}

Funding from the Supersonics Project of the NASA Fundamental Aeronautics Program is gratefully acknowledged. The authors would also like to thank Hyun Dae Kim of NASA GRC for acquiring the 16" MFP data.

\section{REFERENCES}

[1] Iek, C., "Calibration of the 15" Mass-Flow Plug in NASA LeRC's 10'x 10' Supersonic Wind Tunnel," Case Western Reserve University EMAE 657 Report, March 19, 1996.

[2] Bean, H. S. (ed), 1971, Fluid Meters: Their Theory and Application, 6th Ed., American Society of Mechanical Engineers, New York.

[3] Rivas, R.A., Jr. and Shapiro, A.H., "On the Theory of Discharge Coefficients for Rounded-Entrance Flowmeters and Venturis," Trans. ASME, Vol.78, No.3, 1956, pp.489-497.

[4] Cotton, K. C., and Westcott, J. C., "Throat Tap Nozzles Used for Accurate Flow Measurements," Journal of Engineering for Power, Transactions of the ASME, October 1960, pp. 247-263.

[5] Smith, R. E., and Matz, R. J., "A Theoretical Method of Determing Discharge Coefficients for Venturis Operating at Critical Flow Conditions," Journal of Basic Engineering, Transactions of the ASME, December 1962, pp. 434-446.

[6] Lahti, D.J. and Hamed, A., "Verification of the Theoretical Discharge Coefficient of a Subcritical Airflow Meter," Journal of Propulsion and Power, Vol.9, No.4, July-August, 1993, pp.615-621.

[7] Keith, J.S., Ferguson, D.R., Merkle, C.L., Peck, C.H., and Lahti, D.J., "Analytical Method for Predicting the Pressure Distribution about a Nacelle at Transonic Speeds," NASA CR-2217, July, 1973.

[8] Harris, J.E., "Numerical Solution of the Laminar, Transitional, and Turbulent Boundary-Layer Equations and Comparison with Experimental Data," NASA TR R-368, August, 1971.

[9] Harris, J.E., and Blanchard, D.K., "Computer Program for Solving Laminar, Transitional, or Turbulent Compressible Boundary-Layer Equations for Two-Dimensional and Axisymmetric Flow," NASA TM 83207, 1982.

[10] Stockman, N.O. and Farrell, C.A., "Improved Computer Programs for Calculating Potential Flow in Propulsion System Inlets," NASA Technical Memorandum TM-73728, July, 1977.

[11] Herring, H.J., "PL2 - A Calculation Method for TwoDimensional Boundary Layers with Cross-flow and Heat Transfer," Dynalysis of Princeton Report Number 65, June 1981.

[12] Towne, C., "Wind-US User's Guide, Version 2.0," Glenn Research Center, NASA/TM-2009-215804, Oct. 2009.

[13] Menter, F.R., "Two-Equation Eddy Viscosity Turbulence Models for Engineering Applications," AIAA Journal, Vol. 32, No. 8, 1994, pp. 1598-1605.

[14] Georgiadis, N., and Papamoschou, D., "Computational Investigations of High-Speed Dual-Stream Jets," AIAA Paper 20033311, May 2003.

[15] Georgiadis, N.J., Rumsey, C.L., Yoder, D.A., and Zaman, K.B.M.Q., "Turbulence Model Effects on Calculation of Lobed Nozzle Flowfields," Journal of Propulsion and Power, Vol. 22, No. 3, 2006, pp.567-575.

[16] Blumenthal, P.Z., 1995, "A PC Program for Estimating Measurement Uncertainty for Aeronautics Test Instrumentation," AIAA Paper 1995-3072, 31st AIAA Joint Propulsion Conference and Exhibit, San Diego, CA. 